\title{
Myocardial perfusion imaging with 99mTc- labeled radiopharmaceuticals: How fast can a stress-rest same-day imaging protocol be completed?
}

\author{
Raymond Taillefer, MD, FRCP, ABNM ${ }^{a, b}$ \\ a Département de médecine nucléaire, Hôpital du Haut-Richelieu, Saint-Jean-sur-Richelieu, QC, \\ Canada \\ b Department of Radiology, Radio-Oncology and Nuclear Medicine, Université de Montréal, \\ Montréal, QC, Canada
}

Received Feb 22, 2016; accepted Feb 22, 2016

doi: $10.1007 / \mathrm{s} 12350-016-0461-z$

See related article, pp. 1314-1327

Since its first clinical introduction in the early 1990s, myocardial perfusion imaging (MPI) with either 99mTc-Sestamibi (Cardiolite) or $99 \mathrm{mTc}-T e t r o f o s m i n$ (Myoview) has been the subject of several types of imaging protocols. ${ }^{1}$ Although various parameters must be taken into consideration before establishing a specific imaging protocol, achievement of the highest overall diagnostic accuracy in the safest way for the patient must remain the major goal. In the actual highly "competitive" healthcare environment, obtaining a diagnosis in the easiest, safest, fastest, and less expensive way has almost become mandatory. Most of the non-nuclear cardiologic diagnostic procedures can be completed within 1 hour while a complete same-day MPI protocol still requires a longer time, no matter if a rest-stress or stress-rest injection sequence is used, ${ }^{2}$ unless a stress-only protocol is performed. Most of the current MPI protocols are modulated from two general parameters: pharmacokinetics of the radiopharmaceuticals and the type of detector-software combination used in the imaging data acquisition and analysis process. In

Reprint requests: Raymond Taillefer, MD, FRCP, ABNM, Département de médecine nucléaire, Hôpital du Haut-Richelieu, 920 Boulevard du Séminaire Nord, Saint-Jean-sur-Richelieu, QC J3A 1B7, Canada; rtaillefer@hotmail.com

J Nucl Cardiol 2017;24:1328-31.

$1071-3581 / \$ 34.00$

Copyright (c) 2016 American Society of Nuclear Cardiology. recent years, researches have been focused on the development of dedicated cardiac systems showing potential benefits such as patient comfort, shorter acquisition time, and/or reduced administered activity. ${ }^{3-5}$ In comparison with the traditional scintillation detector technology, novel detector technology such as pixelated solid-state CZT (an alloy of cadmium telluride and zinc telluride) detectors offers a better energy resolution and a virtually linear count rate response. This new type of detector has demonstrated similar performance as traditional systems, but with significant shorter imaging times or lower administered radioactivity, achieving a reduction in absorbed dose to $1 \mathrm{mSv}$ for a single injection of MPI radiotracer. ${ }^{6}$ Another variation from traditional design is the upright position offered in some dedicated systems. ${ }^{7}$ Other camera systems offer a rotating chair instead of a rotating gantry or special detectors to allow for optional $\mathrm{x}$-ray-based attenuation correction. Another upgraded technology uses a conventional multi-purpose SPECT system with a cardiofocal collimator and a specific reconstruction algorithm allowing for reduction in acquisition times with no loss in image quality.

The other variable to consider in modifying an imaging protocol is the data from pharmacokinetics of MPI radiotracers. Although their chemical structures are quite different, ${ }^{8}$ 99mTc-sestamibi and $99 \mathrm{mTc}$-Tetrofosmin share many common characteristics, the most important one being the lack of clinically significant myocardial redistribution (with a myocardial half-life of approximately $4 \mathrm{~h}$ ). Pharmacokinetic data represent one of the major parameters to consider in the design of an imaging protocol with MPI radiotracers. The ideal imaging time should reflect the best compromise 
between high myocardial count rate and the lowest surrounding organ uptake (mostly lung, liver and gallbladder). Biodistribution studies in humans ${ }^{9,10}$ have shown that the initial pulmonary activity of both radiotracers was negligible after stress and rest administration and that the myocardial uptake of 99Tc-sestamibi at stress (expressed as \% of injected dose) was $1.5 \pm 0.4$ (at 5 minutes) and $1.4 \pm 0.3$ (at 60 minutes) and for 99 mTc-Tetrofosmin $1.3 \pm 0.3$ (at 5 minutes) and $1.1 \pm 0.2$ (at 60 minutes), while the myocardial uptake of 99Tc-sestamibi at rest was $1.2 \pm 0.4$ (at 5 minutes) and $1.0 \pm 0.4$ (at 60 minutes) and for $99 \mathrm{mTc}$-Tetrofosmin $1.2 \pm 0.3$ (at 5 minutes) and $1.2 \pm 0.4$ (at 60 minutes). Since the major route of excretion of both radiotracers is through the hepatobiliary tree, the second most important organ to consider in the imaging protocol is the liver and the gallbladder. The liver uptake of 99Tc-sestamibi at stress was $5.9 \pm 2.9$ and $2.4 \pm 1.6$ (at 5 and 60 minutes, respectively) and for $99 \mathrm{mTc}$-Tetrofosmin $3.2 \pm 1.9$ and $1.0 \pm 0.5$ (at 5 and 60 minutes, respectively), while the liver uptake of 99Tc-sestamibi at rest was $19.6 \pm 7.1$ and $5.6 \pm 1.6$ (at 5 and 60 minutes, respectively) and for $99 \mathrm{mTc}$-Tetrofosmin $7.5 \pm 1.7$ and $2.1 \pm 2.0$ (at 5 and 60 minutes, respectively).

Considering the potential variability of hepatic clearance through the biliary tree (variable hepatobiliary transit time), and possible related "subdiaphragmatic contamination," and considering that there is no clinically relevant myocardial redistribution within few hours after its administration, it was initially recommended to wait approximately 60-75 minutes after 99mTc-sestamibi intravenous injection before starting the image acquisition. ${ }^{11,12}$ Subsequently, different approaches have been proposed in order to decrease this relatively long time interval between injection and imaging. ${ }^{1}$ Although initially the liver uptake was seen as a potential cause of image artifacts, it was soon recognized that the hepatobiliary excretion would more likely affect the imaging protocols. Some "recipes" such as the oral administration of water, cream, or fatty meal have been used to increase the biliary excretion. ${ }^{13}$ However, this increased rate of excretion of biliary activity may cause a "collateral damage" such as a rapid increased uptake into the duodenum, which may be positioned in the left upper quadrant of the abdomen or may sometimes reflux into the stomach, which lies immediately adjacent to the inferior wall of the left ventricle, secondary to an entero-gastric reflux, causing a more persistent and more prominent uptake. According to the location, extent, and intensity of this subdiaphragmatic tracer concentration, significant imaging artifacts can be created, causing either an inferolateral wall myocardial perfusion defect ("negative ramp filter', artifact) or a focal artifactual increased inferior wall count density (Compton scattering activity). According to the type of image artifact (false increase or decrease uptake) and its time of occurrence (on the rest or the stress study), this may have a significant clinical impact on the overall diagnostic accuracy of the test. Since this hepatobiliary clearance pattern is variable and hard to predict in a given patient, a unique imaging protocol is likely to be more difficult to apply.

Therefore, since the clinical introduction of $99 \mathrm{mTc}-$ labeled MPI agents, various imaging protocols, including the rest and stress injection sequence and the time interval between intravenous injection and the beginning of image acquisition, have been described in the literature. Published ASNC (American Society of Nuclear Cardiology) imaging guidelines specify the optimal minimum delays for both $99 \mathrm{mTc}$-sestamibi and $99 \mathrm{mTc}-$ tetrofosmin. ${ }^{14}$ For $99 \mathrm{mTc}$-sestamibi, the recommended minimum delays between radiotracer administration and imaging are 15-20 minutes for exercise, 45-60 minutes for rest, and 60 minutes for pharmacologic stress, and for $99 \mathrm{mTc}$-tetrofosmin the minimum delays are 1015 minutes for exercise, 30-45 minutes for rest, and 45 minutes for pharmacological stress. The differences between the two radiotracers are related to the difference in their respective biological characteristics. Considering the "standard" image acquisition time, the traditional time interval between the injection and images, and the time interval between the completion of the first set of images and the second injection of the radiotracer, a rest-stress or stress-rest injection sequence for a single-day protocol still requires almost 3 to 4 hours to be completed.

In this current issue of the Journal, Katsikis et al. ${ }^{15}$ report the results of a prospective study comparing early (15 minutes post i.v) and late (45 minutes post i.v) MPI with $99 \mathrm{mTc}$-tetrofosmin. They evaluated the feasibility and diagnostic accuracy of a fast imaging protocol with both exercise and pharmacologic vasodilation in a diagnostically challenging patient population. Their entire MPI protocol was completed within 150 minutes. Using standard SPECT gamma cameras and acquisition, they showed a similar diagnostic accuracy and overall image quality for both early and late imaging. They confirmed results from previous studies evaluating the same issues. ${ }^{16-18}$ A fast MPI protocol including both rest and stress studies has several clinical implications. On the practical side, it offers a shorter waiting times and convenience to the patient and it increases the laboratories efficiency, possibly doing more studies per day. Furthermore, if there is significant patient motion or if technical problems occur, another set of images can be easily repeated. Besides these logistic considerations, there are also the potential diagnostic benefit of reducing 
the time interval between injection and imaging. Some studies have shown increased detection of ischemia using early imaging although this did not translate into a statistically significant difference in diagnostic accuracy, probably related to the relatively small number of patients. Another diagnostic advantage is the detection of post-exercise stunning using early gated SPECT MPI. Mut et al. ${ }^{19}$ evaluated the feasibility and the value of early post-stress imaging (15 minutes) to the traditional delayed imaging (60 minutes) with $99 \mathrm{mTc}$-sestamibi. There was no statistically significant difference in the quality of the images from both sets of post-stress studies. Furthermore, there were no studies considered uninterpretable due to increased subdiaphragmatic radiotracer accumulation. The other endpoint of their study was to use early gated SPECT imaging to detect post-exercise myocardial stunning. Myocardial uptake of perfusion radiotracer at the time of SPECT acquisition represents the relative myocardial perfusion pattern at the time of the tracer injection, but the gated SPECT represents LV function at the time of image acquisition, which can be obtained from 15 to 60 minutes or even more post injection. Therefore, gated SPECT imaging performed earlier after radiotracer administration could potentially detect more post-exercise LV dysfunction. ${ }^{20-22}$ Mut et $\mathrm{al}^{19}$ showed that the change in the LV ejection fraction in patients with ischemia was greater between the rest and the early stress imaging than between the rest and delayed stress studies. Unfortunately, as pointed out by the authors, wall motion or thickening was not assessed nor clinical data on the prognostic value from change in LV ejection fraction obtained.

MPI protocols are not static; on the contrary, according to the physiologic approach used in nuclear cardiology procedures, these protocols are dynamic and must be adapted to most recent validated technologic advances and new data on kinetics. The traditional MPI protocols were based on the knowledge obtained from animal and early human data and were judged to represent the best compromise between high myocardial counts and lower uptake in the adjacent organs, given the type of SPECT gamma cameras and softwares available at that time. It is now obvious that time has come to consider the improvement or modification of MPI guidelines based on new available clinical data. If a complete rest-stress or stress-rest single-day MPI protocol using "standard" detection devices can be shortened to approximately 150 minutes without altering the diagnostic accuracy, and potentially increasing it with detection of more ischemic segments and better detection of post-ischemic stunning as well as transient dilatation of the left ventricle or increased lung uptake, it is conceivable that this entire length of the imaging protocol could be further decreased with new imaging modalities.
Considering that new detectors technology can be used to either decrease the imaging time or the administered activity, or possibly both, it is possible then to consider an acquisition time of less than 5 minutes for each set of images. The only major limitation will be the time interval between the rest and stress radiotracer administration. The ratio between the two doses would then have to be modified in order to overcome the absence of significant radioactive decay between the two injections if the time interval is too short and the possible "myocardial background contamination" from the first study over the second one. Another potentially interesting venue would be to re-consider the use of $99 \mathrm{mTc}$-Teboroxime which was almost simultaneously approved with $99 \mathrm{mTc}-$ sestamibi. The myocardial clearance half-time of 910 minutes, the peak hepatic uptake at 5-6 minutes after injection, the blood pool activity clearance of 1-2 minutes, and scattered activity from liver uptake have challenged investigators to find a clinically useful and optimal imaging protocol with 99Tc-Teboroxime. ${ }^{23}$ Because of these limitations, this radiotracer was not really used in clinical practice. However, given the recent technologic improvement with new detectors, softwares, and scatter correction methods, it would be interesting to re-investigate the clinical potential of $99 \mathrm{mTc}$-Teboroxime or other similar agents. This could even further significantly reduce the entire imaging protocol duration.

Of course, more prospective data and more "headto-head" comparative studies with these new protocols and new imaging modalities will be needed in order to fully validate their diagnostic accuracy and establish adequate and specific normal database for quantitation. It is likely that MPI guidelines will need to be upgraded in a short term future to take into consideration all the recent knowledge in MPI.

\section{References}

1. Wackers FJT. The maze of myocardial perfusion imaging protocols in 1994. J Nucl Cardiol 1994;1:180-8.

2. Taillefer R, Gagnon A, Laflamme L, Grégoire J, Léveillé J, Phaneuf DC. Same day injection of Tc-99m methoxy isobutyl isonitrile (hexamibi) for myocardial tomographic imaging: Comparison between rest-stress and stress-rest injection sequences. Eur J Nucl Med 1989;15:113-7.

3. Garcia EV, Faber TL, Esteves FP. Cardiac dedicated ultrafast SPECT cameras: New designs and clinical implications. J Nucl Med 2011;52:210-7.

4. DePuey EG. Advances in SPECT gamma camera software and hardware: Currently available and new on the horizon. J Nucl Cardiol 2012;19:551-81.

5. Verberne HJ, Acampa W, Anagnostopoulos C, et al. EANM procedural guidelines for radionuclide myocardial perfusion imaging with SPECT and SPECT/CT. www.eanm.org/publications/guidelines/2015_07_ EANM_Final_Myocardial_Perfusion_Guidelines. 
6. Einstein AJ, Blankstein R, Andrews H, Fish M, Padgett R, Hayes $\mathrm{SW}$, et al. Comparison of image quality, myocardial perfusion, and left ventricular function between standard imaging and single-injection ultra-low-dose imaging using a high-efficiency SPECT camera: The MILLISIEVERT study. J Nucl Med 2014;55:1430-7.

7. Ben-Haim S, Almukhailed O, Neill J, Slomka P, Allie R, Shiti D, et al. Clinical value of supine and upright myocardial perfusion imaging in obese patients using the D-SPECT camera. J Nucl Cardiol 2014;21:8.

8. Taillefer R. Technetium-99m Sestamibi, in New radiotracers in cardiac imaging: principles and applications. Taillefer-Tamaki: Appleton and Lange; 1999. p. 3-48.

9. Wackers FJ, Berman DS, Maddahi J, et al. Technetium-99m hexakis-2-methoxyisobutyl isonitrile: Human biodistribution, dosimetry, safety and preliminary comparison to thallium-201 for myocardial perfusion imaging. J Nucl Med 1989;30:301-11.

10. Higley B, Smith FW, Smith T, Gemmell HG, Das Gupta P, Gvozdanovic DV, et al. Technetium-99m-1,2-bis(2-ethoxyethyl) phosphino)ethane: Human biodistribution, dosimetry and safety of a new myocardial perfusion imaging agent. J Nucl Med 1993;34:30-8.

11. Taillefer R, Lambert R, Dupras G, Grégoire J, Léveillé J, Essiambre R, et al. Clinical comparison between thallium-201 and Tc99m- methoxy isobutyl isonitrile (hexamibi) myocardial perfusion imaging for detection of coronary artery disease. Eur J Nucl Med 1989;15:280-6.

12. Francheschi M, Guimond J, Zimmerman RE, Picard MV, English RJ, Carvalho PA, et al. Myocardial clearance of Tc-99m hexakis2-methoxy-2-methylpropyl isonitrile (MIBI) in patients with coronary artery disease. Clin Nucl Med 1990;5:307-12.

13. Hurwitz GA, Clark EM, Slomka PJ, Siddiq SK. Investigation of measures to reduce interfering abdominal activity on rest myocardial images with 99mTc-sestamibi. Clin Nucl Med 1993; 18:735-41.

14. Henzlova MJ, Cerqueira MD, Hansen CL, Taillefer R, Yao SS. ASNC imaging guidelines for nuclear cardiology procedures: Stress protocols and tracers. J Nucl Cardiol 2009;16:331-44.
15. Katsikis A, Theodorakos A, Kouzoumi A, Kitziri E, Georgiou E, Koutelou M, et al. Fast myocardial perfusion imaging with ${ }^{99 \mathrm{~m}} \mathrm{Tc}$ in challenging patients using conventional SPECT cameras. J Nucl Cardiol 2016. doi:10.1007/s12350-016-0431-5.

16. Taillefer R, Lambert R, Bisson G. Myocardial technetium-99mlabeled sestamibi single-photon emission computed tomographic imaging in the detection of coronary artery disease: Comparison between early ( 15 minutes) and delayed (60 minutes) imaging. J Nucl Cardiol 1994;1:441-8.

17. Giorgetti A, Rosi M, Stanislao M, Valle G, Bertolaccini P, Maneschi A, et al. Feasibility and diagnostic accuracy of a gated SPECT early-imaging protocol: A multicenter study of the Myoview imaging optimization group. J Nucl Med 2007;48:1670-5.

18. Philippe L, Merino B, Blaire T, Bailliez A, Casset-Senon D, Levy $\mathrm{M}$, et al. Tetrofosmin early time gated post-stress single=photon emission computed tomography imaging: Feasibility and potential benefits. J Nucl Cardiol 2011;18:62-72.

19. Mut F, Giubbini R, Vitola J, Lusa L, Sobic-Saranovic D, Peix A, et al. Detection of post-exercise stunning by early gated SPECT myocardial perfusion imaging: results from the IAEA multi-center study. J Nucl Cardiol 2014;21:1168-76.

20. Johnson LL, Verdesca SA, Aude WY, Xavier RC, Nott LT, Campanella MW, et al. Postischemic stunning can affect left ventricular ejection fraction and regional wall motion on poststress gated sestamibi tomograms. J Am Coll Cardiol 1997;30: 1641-8.

21. Toba M, Kumita S, Cho K, Ibuki C, Kumazaki T, Takano T. Usefulness of gated myocardial perfusion SPECT imaging soon after exercise to identify postexercise stunning in patients with single-vessel coronary artery disease. J Nucl Cardiol 2004;11:697703.

22. Sciagra R, Sotgia B, Dona M, Pupi A. Influence of the postexercise acquisition delay on the detection of functional abnormalities in sestamibi-gated SPECT. J Nucl Cardiol 2007;14:334-40.

23. Narra RK, Feld T, Nunn AD. Absorbed radiation dose to humans from Technetium-99m-Teboroxime. J Nucl Med 1992;33:88-93. 